\title{
A Study on the Histological and Biochemical Effects of Long-Term Exposure of 4G LTE Radiation Emitted by Mobile Phone on The Liver of Wistar Rats
}

\author{
Arjilli Vamsy ${ }^{1 *}$, V. Sathia Lakshmi ${ }^{2}$ and T. Satya Prakash Venkatachalam ${ }^{3}$ \\ 'Assistant Professor, Department of Anatomy, GSL Medical College Rajahmundry, NH16, Lakshmi Puram, \\ Rajahmundry - 533296, Andhra Pradesh, India; bondu.vamsi@gmail.com \\ 2Professor \& Head of Department, Department of Anatomy SSMCRI, Ammapettai, Chengalpet Taluk, \\ Kancheepuram District, Nellikuppam - 603108, Tamil Nadu, India \\ ${ }^{3}$ Professor \& Head of Department, Department of Pathology, GSL Medical College Rajahmundry, \\ NH16, Lakshmi Puram, Rajahmundry - 533296, Andhra Pradesh, India
}

\begin{abstract}
Every modern technology has two faces. On the brighter side, the mobile phone has become the most essential part of modern life and is playing a very vital role in the life of the human being from womb to the tomb. Most of the people are engaged with them totally to thrive their daily needs .On the other side they are harmful to the human life as they produce thermal and non thermal stress and they can also be carcinogenic and may lead to neural disorders on long run. According to a research they even effect the environment by producing the non-ionising radiation. There were studies evidencing extinction of some species of birds. Most of the earlier studies were based on 2G and 3G radiation effects, very few had given an account of the effects of $4 \mathrm{G}$ mobile phone radiation so in this study $4 \mathrm{G}$ mobile phone with band width 800-2700 MHZ is used for $96 \mathrm{~min} /$ day for 6 months by means of an WhatsApp video call on the Wistar male rats and their effects on the liver were found to be that there causes an inflammation ranging from mild to moderate, congestion of the vessels, Kupffer cell granuloma histologically and biochemical there showed a increase in the levels of Total bilirubin, Direct bilirubin, SGPT\& AIKP with a decrease in the levels of SGOT. The results display that 4G mobile radiations may have a adverse effects on the liver.
\end{abstract}

Keywords: Effects, Liver, Mobile Phone, Radiation, Wistar Rats

\section{Introduction}

The mobile phone has become the most essential part of modern life and is playing a very vital role in the life of the human being from womb to the tomb. Most of the people are engaged with them totally. People wake up to the alarm set in their mobile phones, they depend on the same for morning news, messages, mails, WhatsApp chats, video calling, you tube to see the cooking recipes and to sort out the locations for their journeys. Mobile phone has become a necessity in our daily lives. For children it is a gadget which engages them and also an educational tool. It is ruling the world in all means. Though very little is known about the side effects of exposure to Mobile Phone Radiation (MPR), research has postulated that prolonged use of mobile phone can cause changes in the blood parameters, microscopic changes and may lead to physical and mental stress including fatigue, sleep disturbance ${ }^{1,3,4}$. Mobile phone is a device that produces non-ionizing radiation and causes thermal and non thermal stress ${ }^{6}$.

*Author for correspondence 
Children are potentially at a greater risk than adults for developing brain cancer since their nervous system is still growing and hence more prone to get cancer.

In the present study $4 \mathrm{G}$ LTE mobile phone has been used with a band width of $800-2700 \mathrm{MHz}$. 4G LTE means "Fourth-generation long term evolution," LTE being a type of $\mathbf{4 G}$ electromagnetic waves that facilitate the fastest connection for a mobile internet experience up to 10 times faster than $3 \mathrm{G}$ with a SAR value of $1.6 \mathrm{~W} /$ $\mathrm{kg}$ for $10 \mathrm{~g}$ of tissue . It uses about 4.5-5.0 MB of data on an average if it is used for $1 \mathrm{~min}$ on a WhatsApp video call. Earlier studies had given that there were alterations in the proliferation of hepatocarcinoma cells in the liver due to radiations of the mobile phones ${ }^{15}$. According to the news of UK a study was found to be that $26 \%$ of all car accidents were caused by a driver using cellphone while he is drving ${ }^{16}$. More studies are required to know the exact effects of mobile phone radiation on human life. In this study, effects of MPR on the livers of Wistar rats has been studied by assessing the histological and biochemical changes.

Abbreviations: Mobile Phone Radiation (MPR), SGOT: Serum Glutamic Oxaloacetic Transaminase, SGPT: Serum Glutamic Pyruvic transaminase, ALKP: Alkaline Phosphatase. MWR: Micro Wave Radiation.

\section{Material and Methods}

The presented study was done at GSL Medical College, Rajahmundry after obtaining permission from the Institutional Animal Ethical Committee, GSL Medical College, Rajahmundry, Andhra Pradesh. 108 male Wistar rats of 30 days old were obtained from M/S Mahaveera Enterprises, Hyderabad. All the animals were housed in the central animal house in GSL Medical College in sterile environment. The animals were maintained at an ambient temperature of $24 \pm 2{ }^{\circ} \mathrm{C}$ and fed on standard pellet diet and water ad libitium, the animals were maintained for a total period of 6 months. The light was maintained in the room for them to get 12 hours of light and 12 hours of dark. The animals were assigned under 3 groups; 36 animals in each group which were maintained in 6 cages per group. A branded mobile phone with $4 \mathrm{G}$ SIM with a SAR value of $1.6 \mathrm{~W} / \mathrm{kg}$ was kept in the rat cage which was the recommended value by the ICNIRP guidelines. The RF meter was kept in the cage to ensure the required frequency of radiation and to substantiate the dosimetry with model ED-65 Cornet Electro Smog meter with detection range $100 \mathrm{MHz}-6 \mathrm{GHz}$. The RF meter was kept at 9 different places all over the cage and the readings were measured. The $96 \mathrm{~min} /$ day for 6 months continuously was considered as long term radiation. Animals in first group [E] were exposed to $4 \mathrm{G}$ mobile phone radiation by a WhatsApp video call every day from another mobile phone of the same brand for 96 min duration, assuming that a person uses mobile phone at least for 4 minutes per half hour in a day for 12 active hours it is going to be 96 $\mathrm{min} /$ day. The other group was control group [C] without radiation exposure, the third group was sham expose (S). Every month 6 animals from the C, S group and $\mathrm{E}$ group were sacrificed by giving the ether anesthesia, 2 $\mathrm{ml}$ of blood was collected through cardiac puncture for carrying out liver function tests i.e., Total Bilirubin, Direct Bilirubin, SGOT, SGPT, ALKP and livers were removed for histopathological examination.

The liver tissues were washed in normal saline and were fixed in $10 \%$ Formal-Saline. Tissues were processed in the histopathology laboratory as per standard operating protocol. Sections were stained using Hematoxylin and Eosin (H\&E) stain. Sections were examined in detail and microscopic findings were compared among the control, sham, $4 \mathrm{G}$ groups. The findings were given Histological Activity Index (HAI) Knodell scoring. The biochemical readings were also analysed among the 3 groups.

Statistical analysis: Analysis of data was done by one way analysis of variance (ANOVA) using SPSS software (Statistical package of social sciences) with Software version 20 for windows. The results were expressed as differences of mean. A level of $P$ value less than 0.05 was considered to be significant.

\section{Observations}

Histological Observations: The following parameters like change in shape and size of the nucleus of hepatocytes, hepatocyte necrosis, hepatocytes apoptosis, formation of Kupffer cell granulomas and periportal, portal and lobular inflammation were noted. Blood vessels were also observed for changes like constriction, dilatation and congestion. (Picture 1,2).

Month wise changes in the liver parenchyma in comparison with control group were studied. The details are as follows.

$1^{\text {st }}$ month: Mild periportal inflammation involving $1 / 3^{\text {rd }}$ of the portal tracts with lymphoplasmacytic cells 


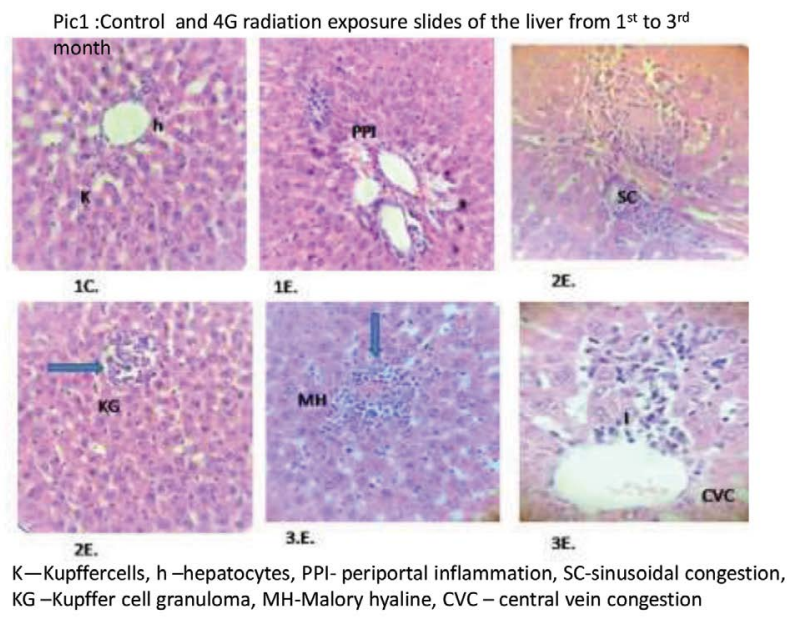

1. C: $1^{\text {st }}$ Month Control, 1. E $1^{\text {st }}$ Month Exposure, 2. E: $2^{\text {nd }}$ Month Exposure, 3. E: $3^{\text {rd }}$ Month Exposure

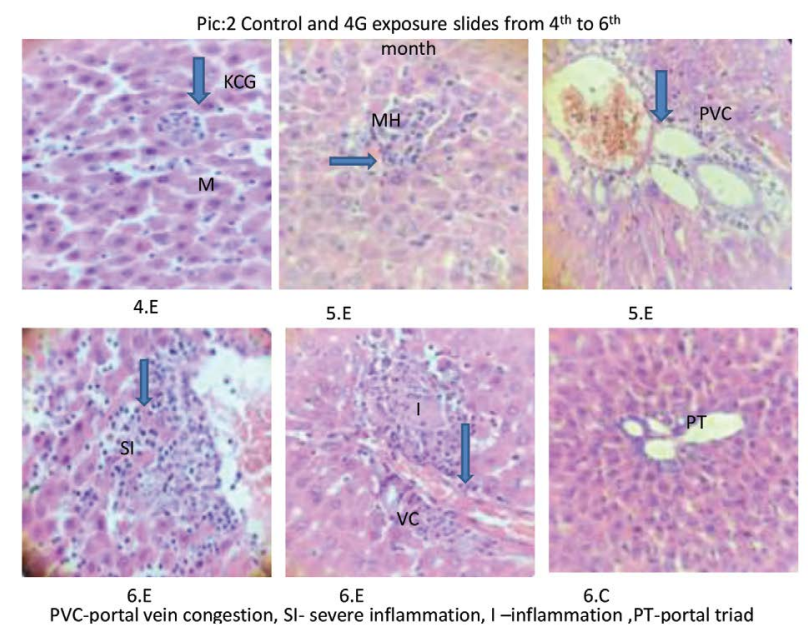

4. E: $4^{\text {th }}$ Month Exposure, 5. E : $5^{\text {th }}$ Month Exposure, 6. E: $6^{\text {th }}$ Month exposure, 6. C: $6^{\text {th }}$ Month Control

and neutrophil, spillage of inflammatory cells into the adjacent hepatocytes, necrosis and apoptosis of hepatocytes. Apoptotic cells showed dense condensed eosinophilic cytoplasm and small pyknotic nuclei. There is random clustering of the Kupffer cells surrounding single cell necrosis.

$2^{\text {nd }}$ month: There is mild inflammation of random portal triads (Portal triaditis), congestion of sinusoids and central vein; and increase in the number of Kupffer cells. Lobular inflammation is also present, with formation of Kupffer cell granulomas around the necrosed hepatocytes. No significant changes in the nuclear size seen.

$3^{\text {rd }}$ month: The hepatocytes showed feathery degeneration of cytoplasm and nuclei were vesicular.
Cytoplasm of the hepatocytes was bubbly or vacuolar in appearance. At the periphery (Zone 1) random hepatocytes show degeneration with clumping of cytoplasm i.e., Mallory hyaline, seen. Mild to moderate inflammation of the portal tracts was observed. There is congestion of central veins surrounding which are seen as lymphocytes and aggregates of Kupffer cells.

$4^{\text {th }}$ month: Moderate inflammation in periportal and sinusoidal areas observed. There is an increase in the number of Kupffer cells, at places these collections forming granulomas around the apoptotic hepatocytes.

$5^{\text {th }}$ month: Severe inflammation in periportal areas, dilation of portal vein radicals with stasis of blood in central vein causing congestion, was seen. Kupffer cell hyperplasia has been observed.

$6^{\text {th }}$ month: Piece meal necrosis of periportal hepatocytes is observed at places occupying more than $50 \%$ of the portal areas, and also features of early degeneration of hepatocytes in the form of shrinkage and dense eosinophilia of the cytoplasm. Apoptotic cells show pyknotic nucleus with dense cytoplasm. There was focal dense collection of the inflammatory cells in $>2 / 3^{\text {rds }}$ of the periportal area. area (Pic. 1, 2). It has been observed that from the first month to sixth month there was a gradual change in the shape of the nucleus from vesicular to pyknotic, increase in number of inflammatory cells in the liver parenchyma, both in portal and lobular areas, increase in the congestion of central vein and the sinusoids. In the sixth month there is more number of lymphoid cells in the sinusoids along with Kupffer cell granulomas. These changes were observed in about $60-90 \%$ i.e. 3-5 animals per month in the radiation exposure group.

Table 1. Bar diagram showing number of animals, month-wise, exhibiting morphological changes in the liver on exposure to mobile phone radiation in comparision to control groups

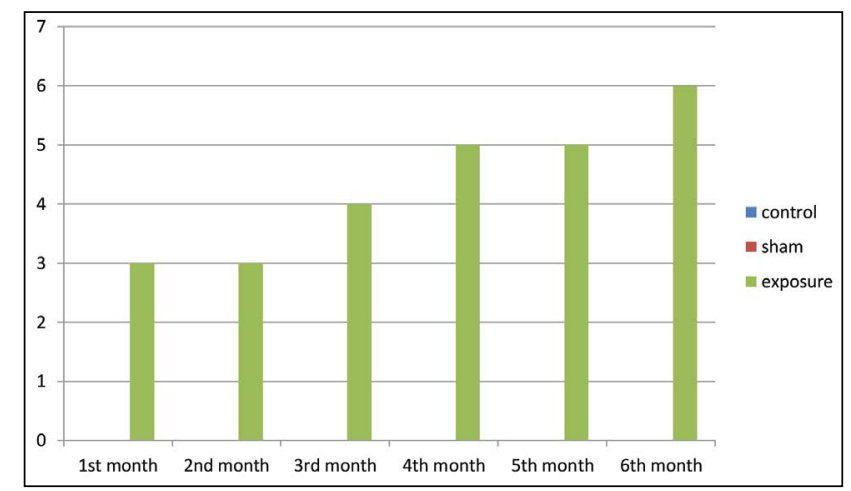


Table 2. Knodell's HAI for Numerical Scoring of Liver Biopsy Specimens

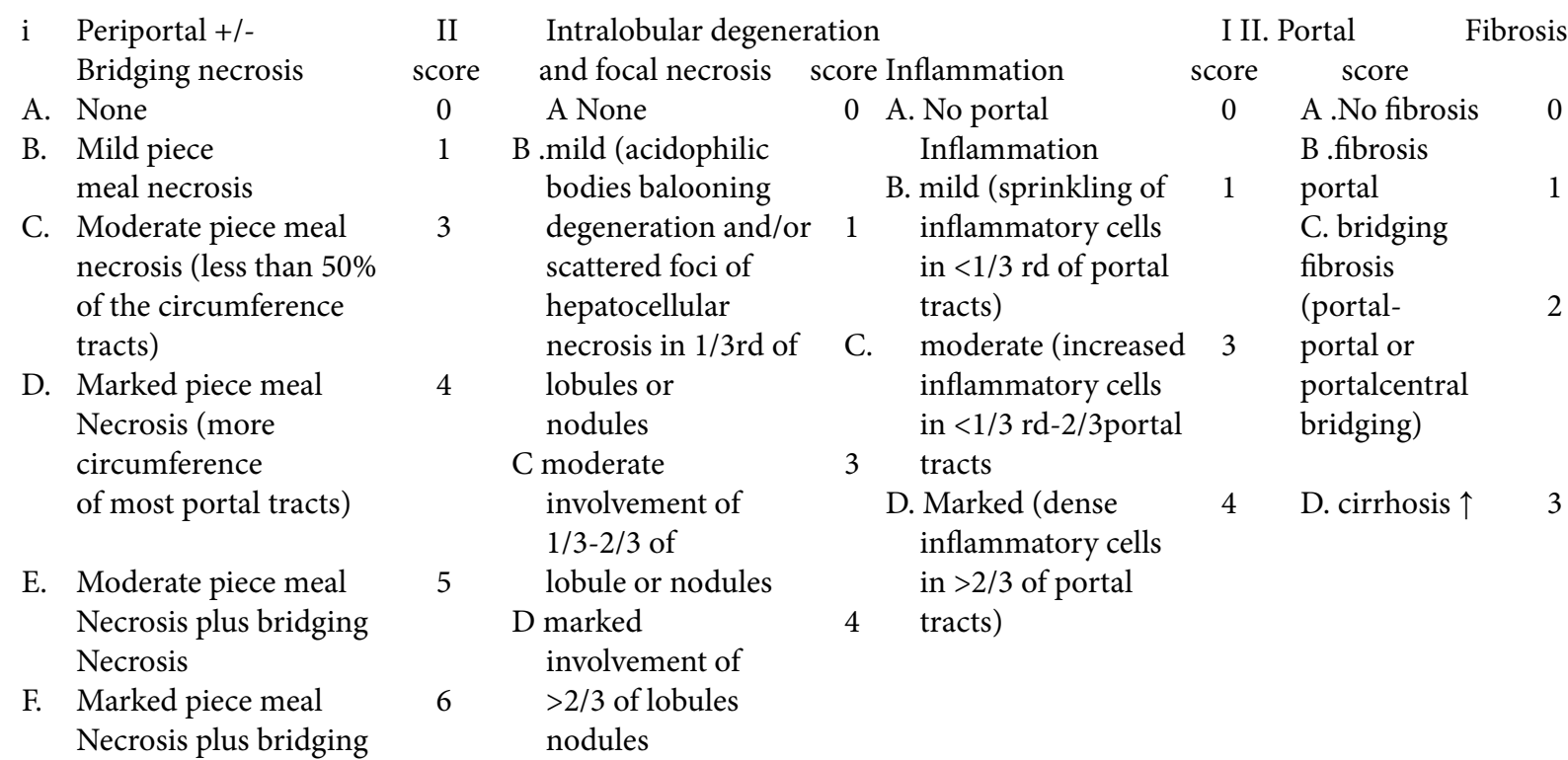

G. Multi lobular necrosis 10

(Table 1.) However no significant histological changes were observed in the control group and sham exposed of all 6 months.

Knodell Histology Activity Index (HAI) though prescribed for progressive assessment of Chronic active hepatitis cases in humans, was taken as a guideline for assessment of histological activity and numerical scoring was done accordingly.

In the present study the Knodell scoring is as follows:

Biochemical observations: The comparison of mean values of control, sham exposed and $4 \mathrm{G}$ exposure groups were considered. There was a gradual raise in the values of the Total bilirubin in the $4 \mathrm{G}$ exposure group compared

Table 3. Knodell score from $1^{\text {st }}$ to $6^{\text {th }}$ month exposure group

\begin{tabular}{|c|c|c|c|c|}
\hline \multicolumn{5}{|c|}{$\begin{array}{l}\text { Month Portal inflammation periportal+/- piece meal } \\
\text { necrosis Interlobular degeneration fibrosis And focal } \\
\text { necrosis }\end{array}$} \\
\hline & Score & Score & Score & Score \\
\hline $1^{\text {st }}$ & 1 & 0 & 0 & 0 \\
\hline $2^{\text {nd }}$ & 1 & 0 & 0 & 0 \\
\hline $3^{\text {rd }}$ & 3 & 0 & 0 & 0 \\
\hline $4^{\text {th }}$ & 3 & 0 & 0 & 0 \\
\hline $5^{\text {th }}$ & 4 & 0 & 0 & 0 \\
\hline $6^{\text {th }}$ & 4 & 4 & 0 & 0 \\
\hline
\end{tabular}

with control and sham from $3^{\text {rd }}$ to the $4^{\text {th }}$ month with significant $\mathrm{p}$ value (Graph 1 ).

In the direct bilirubin there showed a decline in the first 3 months, from $4^{\text {th }}$ month onwards there was a raise in the levels of the direct bilirubin showing significant $\mathrm{p}$ value statistically (Graph 2).

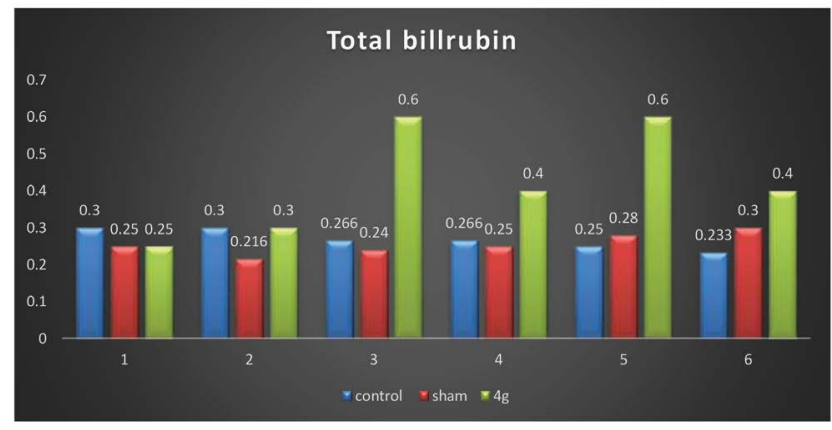

Graph 1. Mean values of Total Bilurubin

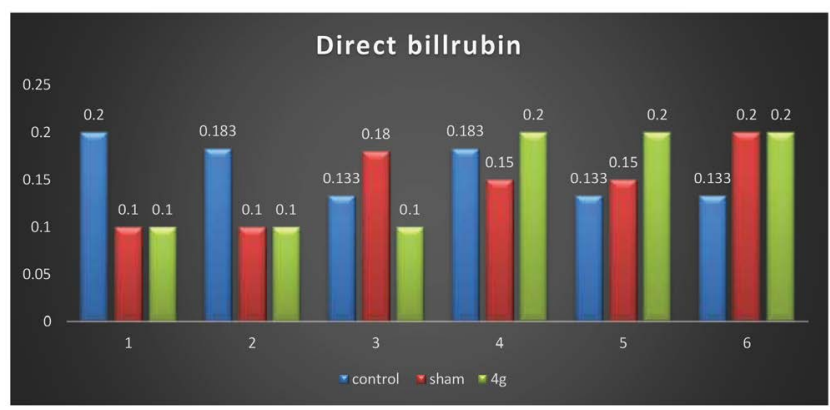

Graph 2. Mean values Direct Bilurubin 
SGOT values had shown a decline from $1^{\text {st }}$ to the $5^{\text {th }}$ month and elevation in the $6^{\text {th }}$ month in comparison with the control and the sham groups, with statistically significant value (Graph 3).

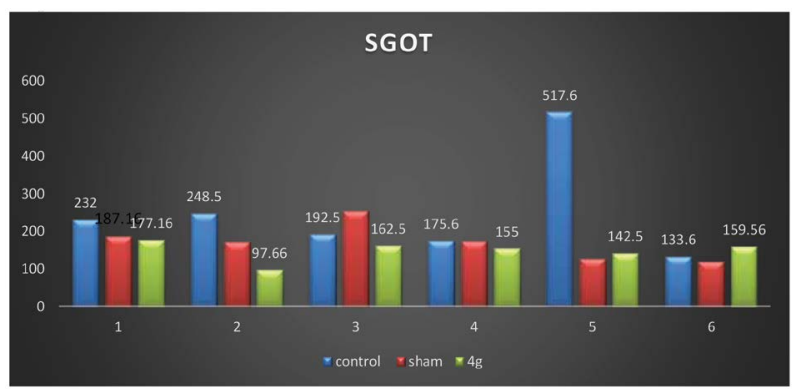

Graph 3. Mean values SGOT

SGPT levels were increased in the first 2 months thereafter showed a drop in the next 4 months compared with the other two groups, having statistically significance (Graph 4).

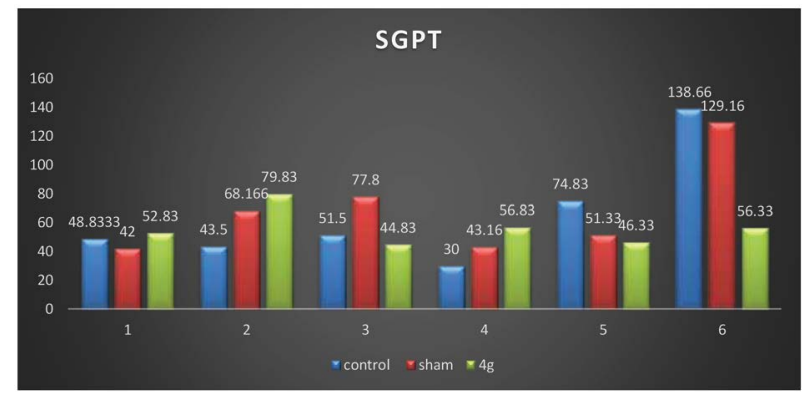

Graph 4. Mean values SGPT

ALKP had shown a drastic elevation in the $1^{\text {st }}$ and $5^{\text {th }}$ months and an overall elevation compared with the control and the sham exposed groups with no significant $\mathrm{p}$ value (Graph 5).

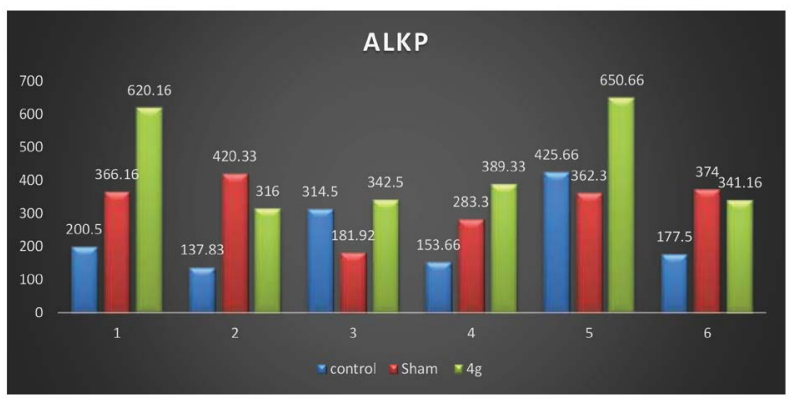

Graph 5. Mean values of ALKP

\section{Discussion}

Nowadays, mobile phones have become a part and parcel of the human life. The present study has been taken up to study whether the mobile phone radiation shows any effect on the human body. Liver being the organ of detoxification in the body, through hepatocytes and also has Kupffer cells which are a part of the defence mechanism of the body. In this study, significant changes were observed in liver on exposure to mobile phone radiation in the form of Kupffer cell hyperplasia, sinusoidal congestion, central vein congestion and portal vein congestion, necrosis and apoptosis of random hepatocytes, focal portal inflammation with Kupffer cell granuloma formation and portal triad it is of random portal triads and piece meal necrosis of periportal hepatocytes from second month onwards of $4 \mathrm{G}$ exposure. Piece meal necrosis of periportal hepatocytes is seen at the end of the $6^{\text {th }}$ month. In comparison with the control, sham and exposure there was an elevated levels of Total bilirubin, direct bilirubin, SGOT and ALKP. SGPT levels were decreased in comparison with the control and the sham. All these changes represent the cellular damage with extensive inflammation which can be construed as a consequence of exposure to mobile phone radiation.

There were severe fatty vacuolation, dilated portal vein and infiltration by large amount of inflammatory cells within the sinusoids when they had exposed the Wistar rats to a radiation of $900 \mathrm{MHz}$. Similar findings were also observed in same in the present research ${ }^{2}$.

Microwave Radiation (MWR) exposure in rats resulted in increased number of micronuclei and discrete perivenular fatty changes in the liver. Perivenular fatty change were not seen in the present study. They reported an increase in the ALT quoted as a mark to the damage of the liver 4 .

Exposure to $4 \mathrm{G}$ for 40 and $60 \mathrm{~min} /$ day for a period of 60 days, certain histopathological findings like portal vein and central vein congestion with their enlargement, mononuclear cellular infiltration surrounding the bile duct and hepatic artery and sinusoidal dilatation were seen. In the present study, similar changes were seen corroborating the effect of $4 \mathrm{G}$ radiation 7

There was moderate hyperaemia dilatation of liver sinusoids, and small inflammatory foci in the centre of liver lobules but no changes in the structure of the 
hepatocytes and the changes were classified as moderate. They also reported that there was occasional hepatocyte necrosis. These changes are in firm consistency with present work ${ }^{11}$.

E.M. Brunts Knodell scoring had showed that there was a considerable damage was produced in the liver tissue after the $4 \mathrm{G}$ mobile phone radiation ${ }^{5,10,20}$.

Similar study to ours was done by in mice using $3 \mathrm{G}$ radiation and similar histological changes were seen in the present study also ${ }^{13,14}$.

An increase in the levels of ALT and AST indicating a damage to the liver structure and the functioning ${ }^{17}$.

A rise in the body temperature, damage to the hepatocytes and liver damage depending on the intensity of the radiation and increase in ALT AST were also mentioned Except for rise in the body temperature which was not recorded in the rats exposed to MPR in this present study, other features observed in that study like liver cell damage had also been encountered in this study along with raise in SGPT, SGOT ${ }^{18,19}$.

Exposure to mobile phone radiation for duration of 2 hours for a period of 2 months can lead to inflammation in the liver, increased levels of ALT marker of the hepatocellular destruction. Similar, observations were seen in this present study which is consonance with their observations $^{21}$.

$900 \mathrm{MHZ}$ of mobile phone radiation for $8 \mathrm{~h} /$ day for 2 months had increased the levels of total bilirubin, our findings with $2700 \mathrm{MHZ}$ of radiation for $96 \mathrm{~min} /$ day for 6 months had also shown a raise in the levels ${ }^{22}$.

Though there were studies in which evidence of carcinomatous changes and oxidative stress produced by the release of free radicals due to radiation was reported, no such changes were observed in the present study ${ }^{11,12,19}$.

\section{Conclusion}

This study finds evidence of inflammation of the liver histologically with damage to hepatocytes and also changes in the vascular structure, and added by altered levels of liver function tests on exposure to MPR which were also a suggestive of inflammation of the liver. The changes were found to be progressive and directly proportional to the length of exposure to MPR. With all the parameters being standardized and the rats were kept in sterile environment, the changes can be construed as a consequence of MPR. Hence it is concluded that long term exposure of Wistar rats to $4 \mathrm{G}$ mobile phone radiation may produce a mild to moderate vascular changes and cellular damage to the hepatocytes and the blood parameters of the liver, that commensurate with the length of exposure to MPR.

\section{References}

1. Abdolmaleki A, Sanginabadi F, Rajabi A, Saberi R. The Effect of Electromagnetic waves exposure on blood parameters. Int J Hematol Oncol Stem Cell Rest of the examination is unchanged from previous evaluation. 2012; 6(2); 13-16.

2. AbuBakr-El Bedwi,Attall F EL-Kott Mohamedsaad and Eman Eid. Effects of Electromagnetic Radiation produced by mobile phones on some visceral Organs of Rat. J. Med. Sci.. 2011; 11(6): 256-2 https://doi.org/10.3923/ jms.2011.256.260

3. AL-Khlawi J, Meo, SA. Association of mobile phone Radiation with fatigue, headache, dizziness, tension and sleep disturbances in Saudi population. Saudi Med J. 2004; 25(6): 732-736.

4. Boris D, Sokolovi D,Kristi D ,Petkovi D, Jovanovi J, Muratovi M .Biochemical and histopathological effects of mobile phone exposure on rat hepatocytes and brain. ActaMedicaMedianae. 2010; 49(1): 37-42.

5. Elizabeth M. Brunt. Grading and staging the histopathological lesions of chronic hepatitis: The Knodell Histological index and beyond Hepatology; 2000: 31(1): 241-2 https:// doi.org/10.1002/hep.510310136

6. Guidelines for limiting exposure to time varying Electric, magnetic and electromagnetic fields (upto 300-4Ghz) health physics. 1998; 74(4): 494-522.

7. Imam Hasan, Mohammad R. Islam. Biochemical and histopathological effects of mobile phone radiation on the liver of Swiss albino mice. Eur J Anat. 2020; 24(4): 257-261.

8. Effects of Mobile Phones on Children's Health [Internet] https://innohealthmagazine.com/. 2021 [cited 17 April 2021]. Available from: https://innohealthmagazine. com/2019/well-being/effects-mobile-phones/

9. International commission on Non-ionizing Radiation protection, Review of the scientific evidence on dosimetry biological effects, epidemiological observations and health consequences concerning exposure to high frequency electromagnetic fields (100khz-300kgh), 2009; Germany ICNIRP161.

10. Knodell R.G, Ishak K.G, Black W.C. Formulation and application of a numerical scoring system for assessing histological activity in asymptomatic chronic active hepatitis. Hepatology. 1981; 1: 431-4 https://doi.org/10.1002/ hep. 1840010511 
11. K, Almasiova V, Cigankova V, Benova K, Racekova E, Martoncikova M. Structural and Ultrastructural Study of Rat Liver Influenced by Electromagnetic Radiation. J. Toxicol. Environ. Health A. 2015; 78(6): 353-3 https://doi. org/10.1080/15287394.2014.979272

12. Merhan Mamdouh Ragy. Effect of exposure and withdrawal of 900-MHz-electromagnetic waves on brain, Kidney and Liver Oxidative Stress and some biochemical parameters in male rats. Electromagn Biol Med. 2015; 34(4). https://doi. org/10.3109/15368378.2014.906446

13. N. Mugunthan, J. Anbalgan, S. Meenachi. Structural changes observed in mice liver due to chronic Exposure of 1900-2200MHZRadiations Emitted from mobile phone. Int. J. Anat Res. 2019; 7(1.3): 6254-https://doi. org/10.16965/ijar.2018.438

14. N. Mugunthan, K. Shanmugasamy, J. Anbalgan, S. Meenachi. Longterm exposure of 900-1900 MHZ mobile phone radiation on mice liver. A histological study. Natl J Clin Anat. 2016; 5(4): 196-2 https://doi.org/10.4103/22774025.297724

15. Ozgur,Elcin; Guler, Gokhur; Kismali, Gurkhan; Seyham,Nescrin. Mobile phone radiation alters proliferation of hepatocarcinoma cells. Cell Biochem Biophys. 2014; 70(2) 983-9 https://doi.org/10.1007/s12013-014-0007-4

16. Ryan Gorman, (March2014) http://www.dailymail.co.uk// news/article-2591148/
17. Samta Sharma, Anjali sharma and Sangeeta Shukla. Effect of Electromagnetic Radiation on Vital Organs in Rats. Octa J. Biosci. 2017; 5(1): 1-4.

18. Sani A, Labaran MM,Dayyabu B. Effects of Electromagnetic Radiation of mobile phones on heamatological and biochemical parameters in male Albinorats. Eur Exp Biol. 2018; 8(2): https://doi.org/10.21767/2248-9215.100052

19. Shahrbanoo Ghaedi, Kargar Jahromi Hossein, Farzam Mohammad, Azhdari Sara, Mahmoudi Teimourabad Saeid, Bathaee Hamid. Effects of Mobile Phone Radiation on Liver Enzymes in Immature Male Rats. Advances in Environmental Biology. 2013; 7(6): 1133-1137.

20. Scheuer P. J. Changing views on chronic hepatitis, histopathology; 1910: 1-https://doi.org/10.1111/j.1365-2559.1986. tb02455.x

21. Sultan Ayoub Meo, Muhammad Arif, Shahzad Rashied, Sufia Husain, Muhammad M. Khan, Abeer A. Al Masri, Muhammad S. Vohra, Adnan M. Usmani, Ashraf Husain, Abdul M. Al-Drees. Morphological changes induced by mobile phone radiation in liver and pancreas in Wistar albino rats. Eur J Anat. 2010; 14 (3): 105-109.

22. Usikalu M. R., Rotimi S. O. \& Achuka J. A. Effects of 900 $\mathrm{MHz}$ radiofrequency radiation on the rats' liver. Jurnal Teknologi (Sciences \& Engineering). 2016; 78: 6-7: 19https://doi.org/10.11113/jt.v78.9078 